OPEN

SUBJECT AREAS:

NANOWIRES

NANOPHOTONICS AND

PLASMONICS

Received

14 October 2013

Accepted

14 October 2013

Published

31 October 2013

Correspondence and requests for materials should be addressed to M.W. (muwang@nju. edu.cn) or R.-W.P. (rwpeng@nju.edu.cn)

\section{Position-sensitive spectral splitting with a plasmonic nanowire on silicon chip}

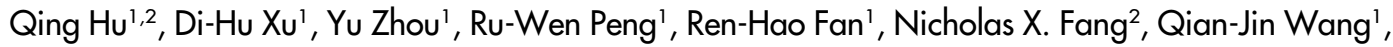 \\ Xian-Rong Huang ${ }^{3} \&$ Mu Wang'
}

\begin{abstract}
${ }^{1}$ National Laboratory of Solid State Microstructures and Department of Physics, Nanjing University, Nanjing 210093, China,
${ }^{2}$ Department of Mechanical Engineering, Massachusetts Institute of Technology, Cambridge, Massachusetts 02139, USA,

${ }^{3}$ Advanced Photon Source, Argonne National Laboratory, Argonne, Illinois 60439, USA.
\end{abstract}

On-chip nanophotonics serves as the foundation for the new generation of information technology, but it is challenged by the diffraction limit of light. With the capabilities of confining light into (deep)

subwavelength volumes, plasmonics makes it possible to dramatically miniaturize optical devices so as to integrate them into silicon chips. Here we demonstrate that by cascading nano-corrugation gratings with different periodicities on silver nanowires atop silicon, different colors can be spatially separated and chronologically released at different grating junctions. The released light frequency depends on the grating arrangement and corrugation periodicities. Hence the nanowire acts as a spectral splitter for sorting/ demultiplexing photons at different nano-scale positions with a ten-femtosecond-level interval. Such nanowires can be constructed further into compact $2 \mathrm{D}$ networks or circuits. We believe that this study provides a new and promising approach for realizing spatiotemporal-sensitive spectral splitting and optical signal processing on nanoscales, and for general integration of nanophotonics with microelectronics.

pectral splitting and imaging have numerous applications varying from optical communication, logical operations, micro-spectrum analyses, to photon sorting or sensing ${ }^{1-6}$. These spectral technologies have been based on traditional filters, mirrors, or interferometers, which are clumsy in size (much larger than the wavelength) and difficult to be integrated into microelectronics ${ }^{7}$. By relying on the silicon-on-insulator (SOI) technology ${ }^{8-10}$, some on-chip spectral devices, such as light in/out couplers ${ }^{11-13}$ and demultiplexers ${ }^{14,15}$, have been successfully developed in the infrared regime, particularly for telecommunication wavelengths. Yet for visible frequencies, the silicon-based technology fails to work efficiently due to the strong inherent absorption of silicon. Consequently, on-chip integration of photonic devices has been limited by their larger-than-wavelength sizes and the weak optical response of silicon ${ }^{16}$. Fortunately, as a product of the interaction between photons and free electrons at the metal-dielectric interface, surface plasmons (SPs) can achieve extremely small mode wavelengths and high localized electromagnetic fields. Hence, plasmonics with (deep) subwavelength characteristics may break the diffraction limit of light, and thus are promising for modulating photons on nanoscales ${ }^{17-19}$ so as to bridge the gap between nanoelectronics and optics ${ }^{20-23}$. In fact, plasmonic structures have shown potential applications as nano waveguides and $\operatorname{circuits}^{24-26}$, nanoantennas ${ }^{27,28}$, submicron dichroic splitters ${ }^{29}$, photonic sorters $^{30}$, and logic gates ${ }^{31}$, etc. However, to date it is still very challenging to make plasmonic devices compatible with the current complementary-metal-oxide-semiconductor (CMOS) technology, mainly due to the large impedance mismatch across the metal-dielectric interface ${ }^{32,33}$. Recently, by introducing hybrid plasmon polaritons $^{34,35}$, researchers have experimentally realized low-loss optical waveguiding on deep subwavelength scales ${ }^{36}$, where the higher optical loss mode, termed as the dielectric-loaded-surface-plasmon-polariton (DLSPP) mode $^{37,38}$, is intentionally converted to a lower hybrid loss mode. But this technique again remains incompatible with the current silicon technology. Therefore, the key issue to advance this field is to explore the possibilities of integrating plasmonic nanostructures on silicon wafers, which is obviously of significant importance for combining nanophotonics and microelectronics and has been attracting intense interest in recent years.

Here we report the first experimental realization of a novel on-chip plasmonic device for position-sensitive spectral splitting of photons with cascading corrugation gratings along silver nanowires atop silicon wafers, where different colors are spatially separated and subsequently released at ten-femtosecond-level intervals. As shown in Fig. 1a, the fundamental mechanisms of this approach are as follows. On the microstructured silver nanowire, the input light is converted into broadband SPs, which then propagate along the wire and are modulated by the plasmonic band gaps of the periodic corrugation gratings. At the junction sites where the spatial periodicity of 


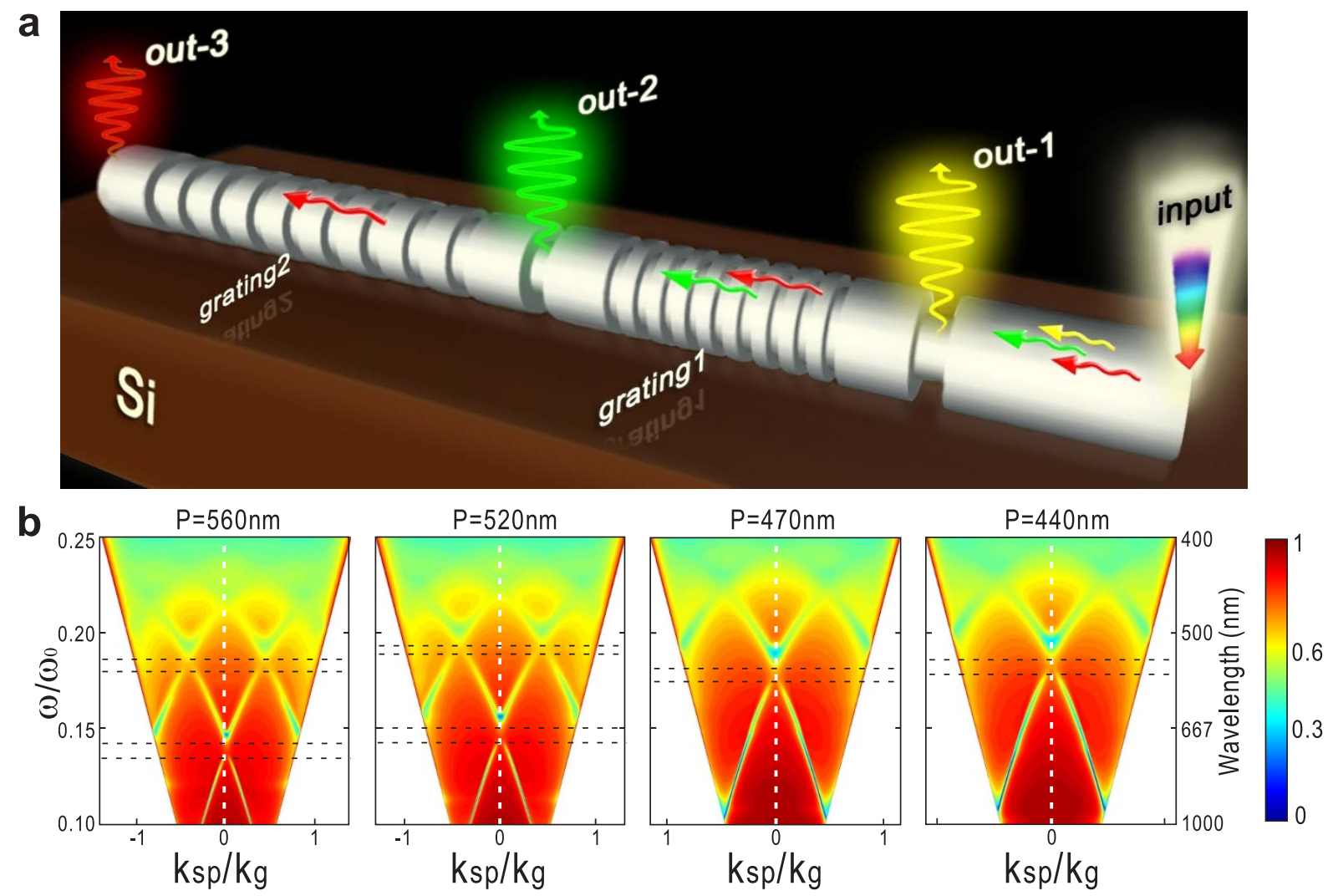

Figure 1 The principle of the plasmonic position-sensitive spectral splitter (PSS) device. (a). Schematic of the plasmonic PSS consisting of two cascading corrugations with different spatial periodicities (marked as "grating1" and "grating2", respectively) for transporting and filtering SPs, and the grooves ("out-1" and "out-2") for exporting SPs. (b). Plasmonic band structures of four (individual) corrugations with periods $P=560 \mathrm{~nm}, 520 \mathrm{~nm}$, $470 \mathrm{~nm}$, and $440 \mathrm{~nm}$, respectively. Here the frequency unit is $\omega_{0}=2 \pi c / a(c$ the speed of light in vacuum and $a=100 \mathrm{~nm})$, the wave-vector unit is $k_{\mathrm{g}}=$ $2 \pi c / P$, and the color bar shows the reflection intensity. The blue-green arcs indicate the dispersion relation of the SPs in the structures. The band gaps appear at the edges of the Brillouin zones marked by the dashed lines. In both the calculations and experiments, the nanowire diameter is always fixed at $170 \mathrm{~nm}$, and the width and depth of the corrugations are both set at $30 \mathrm{~nm}$.

the corrugations is abruptly changed, SPs with a specific wavelength are selectively released. Thus, the structured silver nanowire acts as a position-sensitive spectral splitter (PSS) for photons, as well as a timeof-flight monitor ${ }^{39}$ for femtosecond optical pulses. Such microstructured silver wires can also be constructed further into miniature 2D/ 3D networks/circuits for high-resolution spatiotemporal spectral splitting and imaging ${ }^{40,41}$, optical signal demultiplexing on nanoscales, and many other potential applications of on-chip nanophotonics.

\section{Results}

Concept of a plasmonic nanowire-based spectral splitter atop silicon chip. A smooth silver nanowire without corrugations on a dielectric surface usually supports several types of SP modes, such as guiding modes and leaking modes ${ }^{42,43}$ with a continuous dispersion relation in a wide frequency range ${ }^{44,45}$ (Supplementary Fig. S1a). Thus, it is believed that the silver nanowire on a highpermittivity wafer (silicon, for example) cannot transport regular SP modes ${ }^{46}$. However, a broadband SP mode, which is a hybrid gap mode trapped between the nanowire and the wafer, can be supported (see Supplementary Information). Yet this hybrid SP mode has a small mode area corresponding to large propagation loss, which is still unfavorable for large-distance propagation. To solve this problem, here we intentionally coat an ultrathin $\mathrm{SiO}_{2}$ layer on the silver nanowire and achieve a lower hybrid loss mode $^{36}$. This type of hybrid mode possesses much smaller mode areas $\left(0.06 A_{0}, A_{0}=\lambda^{2} / 4\right)$ and larger propagation lengths (around $15 \mu \mathrm{m}$ at $\lambda=647 \mathrm{~nm}$ ). Consequently, the converted broadband SP modes are able to propagate along the nanowire with much less loss.
Furthermore, by introducing a grating consisting of periodic corrugations on the silver nanowire (shown in Supplementary Fig. S1b), these SP modes can be modulated by the plasmonic band structure that is determined by the spatial periodicity of the grating. Figure $1 \mathbf{b}$ shows the plasmonic band structures of four individual gratings with periodicities of $P=560 \mathrm{~nm}, 520 \mathrm{~nm}, 470 \mathrm{~nm}$, and $440 \mathrm{~nm}$, respect ively. It is obvious that the band gaps appear at $n \frac{\pi}{P}$ (Brillouin zone edges, $n$ being an integer), where the SPs are strongly back-reflected and cannot propagate forward ${ }^{23}$. In our approach, cascading grating segments with different periodicities are constructed on the silver wire, as schematically illustrated in Fig. 1a. Between the cascading grating segments a single groove is introduced, where the localized SPs in the band gap, which possess specific colors, are released back into light by scattering of this groove. In this configuration, the single grooves act as "bus stops" for different colors. Therefore, the periodic corrugation gratings marked as "grating 1" and "grating 2" in Fig. 1a play the role to transport and filter SPs, while the grooves "out-1" and "out-2" act as emitters to release the filtered SPs. Overall, when a beam with mixed wavelengths is introduced into this structured nanowire, broadband SP modes are excited and propagate along the nanowire. Thus, "grating 1" transmits the SPs with frequencies falling within its propagation bands while prohibiting those SPs with frequencies falling in the band gap. Then the prohibited SPs are released at "out-1". Similarly, the SPs that pass through "grating1" but are prohibited by "grating 2" are released at "out-2". The SPs passing through both "grating 1" and "grating 2" are released by "out-3", and so on. With this mechanism, the functionality of subwavelength position-sensitive spectral splitting, demultiplexer or light in/out coupling is realized. 
Experiments for a parallel plasmonic PSS. Firstly, we have fabricated a structured silver nanowire (diameter $170 \mathrm{~nm}$ ) containing two corrugation gratings with periodicities $P=470 \mathrm{~nm}$ ("grating 1 ") and $P=520 \mathrm{~nm}$ ("grating 2"), respectively, as shown in Figs. 2a and $2 \mathbf{b}$. In the middle is a single groove separating the two gratings. The input laser beam is focused on this single groove. The SPs excited by this groove propagate towards the two ends of the nanowire. As mentioned above, since the plasmonic band gaps of the two gratings are different, the output colors at the two ends can be different.

The optical measurements have been carried out with the experimental setup schematically illustrated in Supplementary Fig. S2. The light signals detected at the middle groove and the two ends for different input wavelengths are shown in Figs. $2 d-2 g$. When the wavelength of the input laser is $\lambda=647 \mathrm{~nm}$, both terminals show red bright spots in Fig. 2d. This is reasonable since the wavelength is within the propagation bands of two corrugations with $P=470 \mathrm{~nm}$ and $P=520 \mathrm{~nm}$, as indicated in Fig. 1b. When the input wavelength is changed to $\lambda=568 \mathrm{~nm}$ (Fig. 2e), there is no output from "out-1" (dark) whereas the end "out-2" turns yellow. This indicates that for this wavelength, propagation of the SPs is prohibited in "gratings 1" but allowed in "grating 2", which is again consistent with Fig. 1b. When the input wavelength is further changed to $530 \mathrm{~nm}$, "out-1" turns green and "out-2" becomes dark in Fig. $2 \mathrm{f}$ since SPs corresponding to $\lambda=530 \mathrm{~nm}$ are forbidden in "grating 2". The whole optical spectra of the scattered light measured at the two ends are shown in Figs. $2 c$ (middle and bottom). Compared with the spectrum of a smooth silver nanowire without corrugations (Fig. 2c (top)), the intensity for $\lambda=568 \mathrm{~nm}$ drops dramatically at "out-1", whereas the intensities for $\lambda=514,520$ and $530 \mathrm{~nm}$ attenuate obviously at "out2 ". These experimental data are in good agreement with the calculated normalized transmission (the red lines in Fig. $2 \mathrm{c}$ for continuous wavelength variations).

It should be pointed out that in our experiments, the output intensity of light in the propagation wavelength bands of the corrugation gratings, such as $480-540 \mathrm{~nm}$ and $630-700 \mathrm{~nm}$ in Fig. 2c (middle), maintains the same order of magnitude as that for the smooth nanowire (Fig. 2c (top)). This shows that the transmission efficiency of the SPs on the corrugated structure is high. For example, comparing with the same-length silver nanowire without corrugations, the light going through "grating 2" has the output efficiency around $75 \%$ at $\lambda=647 \mathrm{~nm}$, and $85 \%$ at $\lambda=568 \mathrm{~nm}$, respectively.
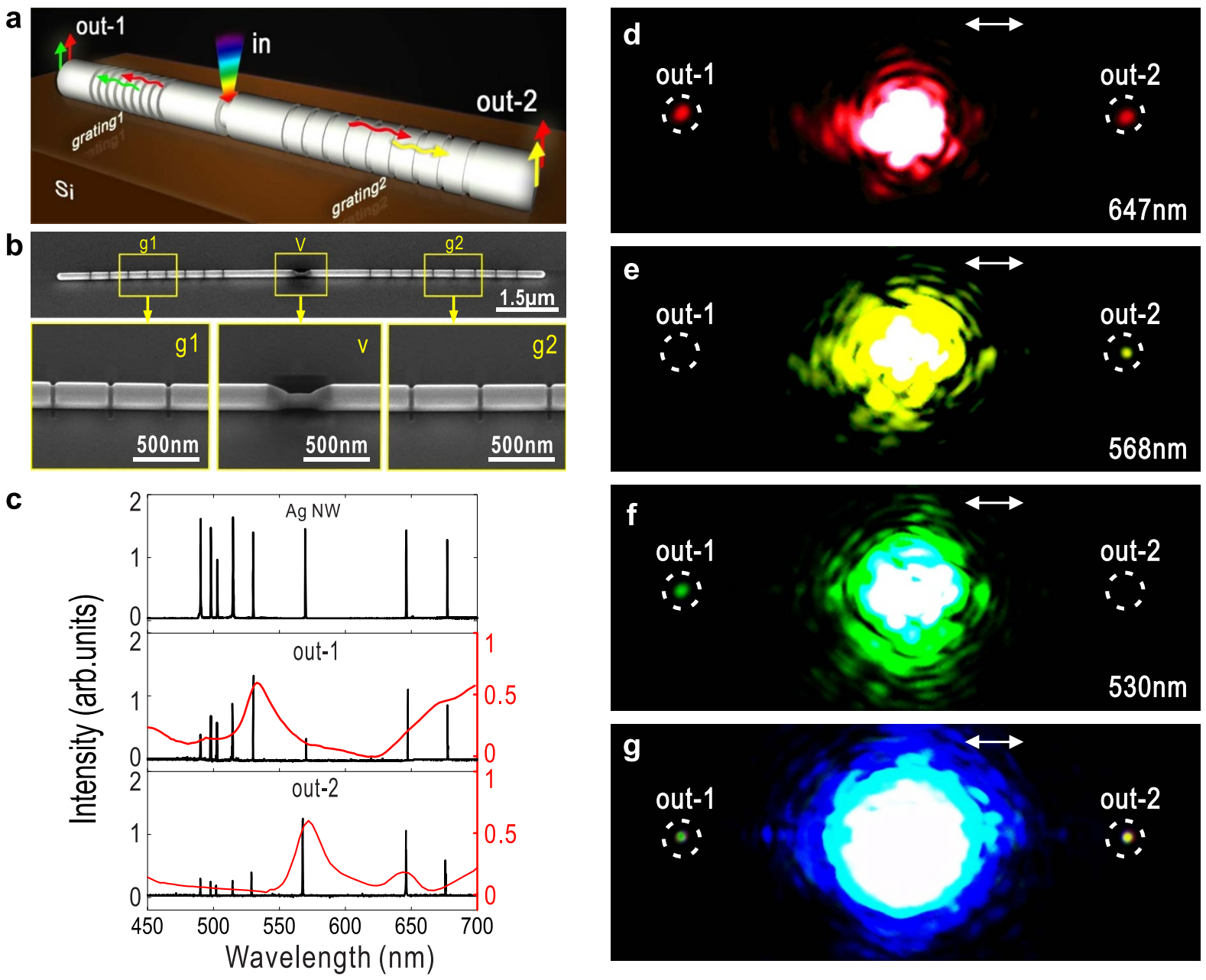

Figure $2 \mid$ Transportation of modulated SPs on the corrugated silver nanowire. (a). Schematic of the structured silver nanowire with two corrugated gratings (with periods $P=470 \mathrm{~nm}$ and $P=520 \mathrm{~nm}$ ) separated by a single groove in the middle that converts the incident beam into SPs propagating towards the two ends of the nanowire. (b). SEM images of the nanowire and the enlarged structure details. The nanowire diameter is about 170 nm; the corrugation period is about $470 \mathrm{~nm}$ for "grating 1", and $520 \mathrm{~nm}$ for "grating 2", respectively. (c). Top: Scattered light spectra measured from the end of a smooth nanowire with no corrugations. Middle and bottom: Experimental (black lines) and calculated (red lines) scattered light spectra from "out-1" and "out-2" of the corrugated nanowire, respectively. (d-f). Emission images of "out-1", the middle groove, and "out-2" for incident wavelengths of $647 \mathrm{~nm}, 568 \mathrm{~nm}$ and $530 \mathrm{~nm}$, respectively. (g). The corresponding emission images for a polychromatic input laser beam, where both ends have output but with different selected colors. The white double-arrow (parallel to the nanowire) indicates the polarization direction of the input beam. 
Experiments for a cascade plasmonic PSS. Now we focus on the propagation of light along the silver nanowire with cascading corrugation gratings in Figs. 3a (also see the schematic of Fig. 1a). This nanowire (170 nm in diameter) possesses two sets of periodic corrugations, one with periodicity $P=470 \mathrm{~nm}$ ("grating 1") and the other with $P=520 \mathrm{~nm}$ ("grating 2"). The two grooves corresponding to "out-1" and "out-2" in Fig. 1a are clearly shown in the SEM images of Fig. 3a (here "out-3" is simply the left end of the nanowire). When a polychromatic laser beam is focused at the input terminal (right most in Fig. 3a), SPs are generated and propagate along the nanowire towards the gratings. When passing through "grating 1", the SP corresponding to $\lambda=568 \mathrm{~nm}$ is forbidden to propagate, so it is scattered at "out-1", resulting in a yellow bright spot at "out-1" in Fig. 3b. The rest of SPs continue to propagate along the nanowire. When they reach "grating 2", the SPs with $\lambda=480-$ $540 \mathrm{~nm}$ are forbidden to propagate. Consequently, they are released at "out-2" as a green bright spot. The remaining SPs are finally exported to the terminal "out- 3 ", where they are released as a red bright spot in Fig. 3b. Figures 3c-e are the optical spectra detected at the three output positions, from which one may identify the strong peaks corresponding to the released SPs in Fig. 3b. These measured spectra agree quite reasonably with the calculated spectra of the red curves in Figs. 3c-e. Some minor discrepancies are mainly due to the fact that the chemically-synthesized nanowire usually does not have strictly round cross-sections or perfectly smooth surface. In fact, the cross-sectional shape of the nanowire indeed affects the confinement of light ${ }^{47}$, i.e., different cross-sectional shapes may change the hybrid optical mode along the wire. More detailed consideration for the discrepancies between experiments and simulations are presented in Supplementary Information. In our experiments, a number of silver nanowire samples with different diameters have been fabricated and optically measured. For instance, we have also made the $260 \mathrm{~nm}$-diameter nanowire with cascade periodic corrugations of $P=440 \mathrm{~nm}$ ("grating 1") and $P=520 \mathrm{~nm}$ ("grating 2") (see Supplementary Information), similar good agreement between experiments and simulations has been achieved. With these data, we conclude that the position-sensitive spectral splitting mechanism has been indeed realized by corrugated silver nanowires on silicon wafers.

It is interesting to characterize further the performance of the onchip plasmonic PSS working at visible frequencies. The spectral selectivity of the splitter is one of the key parameters for practical applications, which can be described by the side-band suppres$\operatorname{sion}^{48,49}$ of the plasmonic structure. Here the side-band suppression is defined as $S_{d B}=-10 \log _{10}\left[I(\lambda) / I_{0}(\lambda)\right]$, where $I(\lambda)$ is the wavelength-dependent intensity collected from the nanowire with corrugated gratings, and $I_{0}(\lambda)$ is the collected intensity from a similar nanowire but without corrugations. For example, the above $170 \mathrm{~nm}$-diameter corrugated silver nanowire (Fig. 3a) possesses the side-band suppression $S_{d B} \cong 34 \mathrm{~dB}$ at $\lambda=568 \mathrm{~nm}$, which may be further improved by changing the diameter of the wire (see the data in Table I). Besides, it is also worthwhile to evaluate the outcoupling efficiency of the released light from the PSS, which can be characterized by $\eta(\lambda)=P(\lambda) / P_{0}(\lambda)$, where $P_{0}(\lambda)$ is the wavelengthdependent power of incident light propagating along the nanowire, and $P(\lambda)$ is the power of the released light. According to Fig. $3 \mathrm{c}$, the outcoupling efficiency of the yellow light $(\lambda=568 \mathrm{~nm})$ can reach about $47 \%$ at "out- 1 " in the $170 \mathrm{~nm}$-diameter device; and it can be further enhanced to about $74 \%$ when the wire diameter is increased to $260 \mathrm{~nm}$. Yet the outcoupling efficiency of green light $(\lambda=$ $520 \mathrm{~nm})$ at "out-2" dramatically attenuates due to the intrinsic loss of SPs. Anyway, based on Table I, one can still expect that within a limited working distance (e.g. $15 \mu \mathrm{m}$ ), high outcoupling efficiency and large side-band suppression can be achieved simultaneously in the plasmonic PSS designs by optimizing the geometry and dimensions of the nanowires. In this way, the performance of the plasmonic a

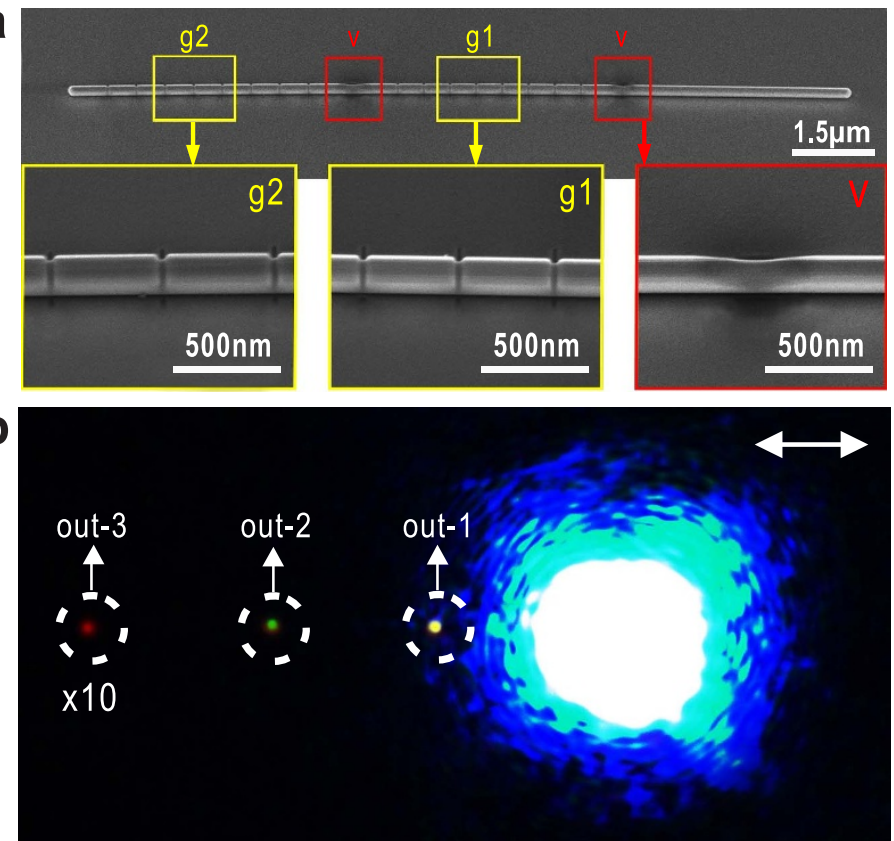

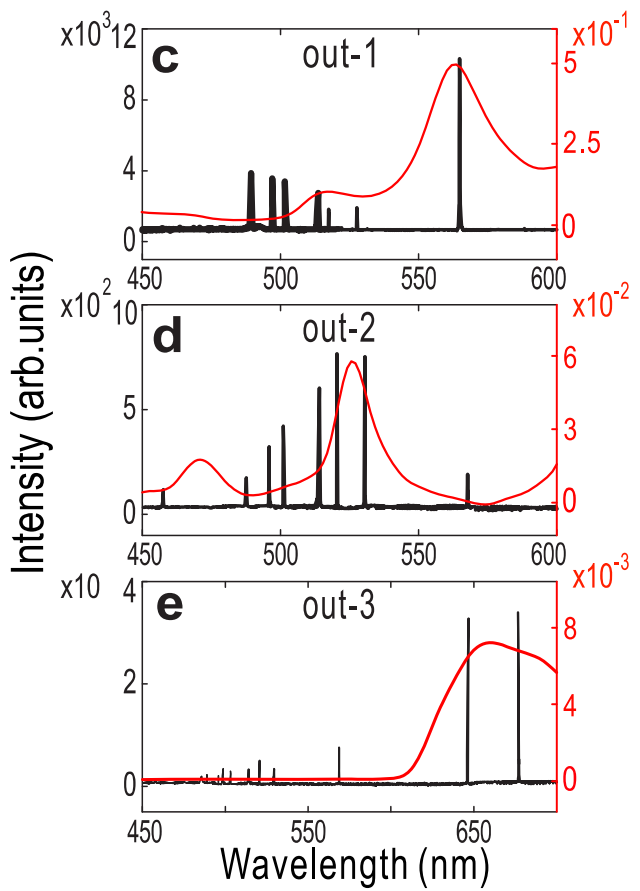

Figure 3 Selective propagation and emission of SPs along a silver nanowire with cascading corrugation gratings. (a). SEM images of the silver nanowire with cascading gratings (corresponding to Fig. 1a) and the enlarged structure details: corrugations with $P=520 \mathrm{~nm}$ (left: grating 2), corrugations with $P=470 \mathrm{~nm}$ (middle: grating 1), and the "bus-stop" groove (right: out-1). The nanowire diameter is about $170 \mathrm{~nm}$. (b). Emission micrograph of the structured nanowire illuminated by a polychromatic laser beam from the input end (rightmost end). The bright spots marked by the dash white circles indicate the light scattered from grooves "out-1", "out-2" and "out-3" (leftmost end). The white double-arrow indicates the polarization direction of the input beam. (c-e). Experimental (black lines) and calculated (red lines) scattered light spectra from "out-1", "out-2" and “out-3", respectively. 
Table I | The performance of the cascade PSS based on two types of silver structured nanowires with different diameter (D) on silicon chip Beam via the wire $\lambda=568 \mathrm{~nm}$ $\lambda=520 \mathrm{~nm}$

\begin{tabular}{|c|c|c|c|c|}
\hline Performance & $D=170 \mathrm{~nm}$ & $D=260 \mathrm{~nm}$ & $D=170 \mathrm{~nm}$ & $D=260 \mathrm{~nm}$ \\
\hline $\begin{array}{l}\text { Side-band suppression } \\
\text { Outcoupling efficiency } \\
\text { Mode area }\left(\mathrm{A}_{0}\right)\end{array}$ & $\begin{array}{c}34 \mathrm{~dB} \\
47 \% @ \text { "out-1" } \\
0.07\end{array}$ & $\begin{array}{c}28 \mathrm{~dB} \\
74 \% @ \text { "out-1" } \\
0.17\end{array}$ & $\begin{array}{c}31 \mathrm{~dB} \\
6 \% \text { @ "out-2" } \\
0.06\end{array}$ & $\begin{array}{c}26 \mathrm{~dB} \\
17 \% @{ }^{\prime \prime} \text { out-2" } \\
0.12\end{array}$ \\
\hline
\end{tabular}

PSS at visible frequencies can match that of non-plasmonic grating couplers (such as SOI waveguides) at infrared wavelengths ${ }^{11-13,50-52}$. On the other hand, with the help of the hybrid SP modes, the plasmonic PSS possesses much smaller mode areas and thus achieves much better confinement of light compared to non-plasmonic devices. For instance, the $170 \mathrm{~nm}$-diameter structured silver nanowire in Fig. 3a has the mode area $A_{\text {pss }} \cong 0.06 A_{0}$ (with $A_{0}=\lambda^{2} / 4$ ), whereas the non-plasmonic waveguides usually have the mode area $A_{\mathrm{dw}} \geq A_{0} / n^{2}$ ( $n$ is the refractive index of the dielectric waveguide material). Given that silicon has high loss at visible frequencies, nonplasmonic waveguides are usually based on other dielectric materials with relatively lower index $n$. Thus for the confinement of visible light, the plasmonic PSS works ten times better than non-plasmonic devices.

Time-domain analysis of a cascade PSS. To understand the transportation process of the SPs in the PSS, we have carried out numerical simulations using the finite-difference time-domain (FDTD) method. Figure 4a shows the calculated electric field distributions around the corrugated nanowire in Fig. 3a for incidence wavelengths of $568 \mathrm{~nm}, 530 \mathrm{~nm}$ and $647 \mathrm{~nm}$, respectively. Apparently, the excited SPs are selectively guided along the nanowire. Around "out-1" in Fig. 4a, the forward propagation of SPs corresponding to $\lambda=568 \mathrm{~nm}$ is blocked, hence the SPs are scattered to the far field.
SPs corresponding to $\lambda=530 \mathrm{~nm}$ pass through "grating 1 " but are blocked around "out-2". In contrast, SPs for $\lambda=647 \mathrm{~nm}$ propagate through both "grating 1" and "grating 2". These FDTD simulations again clearly verify the above results, i.e., different colors are spatially separated along the nanowire due to the microstructures. It should be noted that different colors are released chronologically at 15femtosecond-or-so intervals, as shown in Figs. $4 \mathbf{b}-\mathbf{d}$. Therefore, the structured nanowire simultaneously act as a time-of-flight monitor for femtosecond optical pulses in addition to its function of nanoscale position-sensitive spectral splitting.

\section{Discussion}

It is worthy to emphasize that the present plasmonic PSS possesses the following characteristics. First, the plasmonic nanowire with corrugations can realize on-chip spectral splitting in the visible frequency band. For example, when white light passes through the cascade PSS in Fig. 3a, the outcoupling efficiency of yellow light $(\lambda$ $=568 \mathrm{~nm}$ ) can reach $47 \%$, and meanwhile the side-band suppression can reach about $34 \mathrm{~dB}$. By further optimization of the wire geometry and dimension, the spectral splitting performance of the plasmonic PSS at visible frequencies can match that of non-plasmonic grating couplers (such as SOI waveguides) at infrared wavelengths $s^{51,52}$. For comparison, SOI waveguides fail to work efficiently at visible frequencies because of the strong inherent absorption of
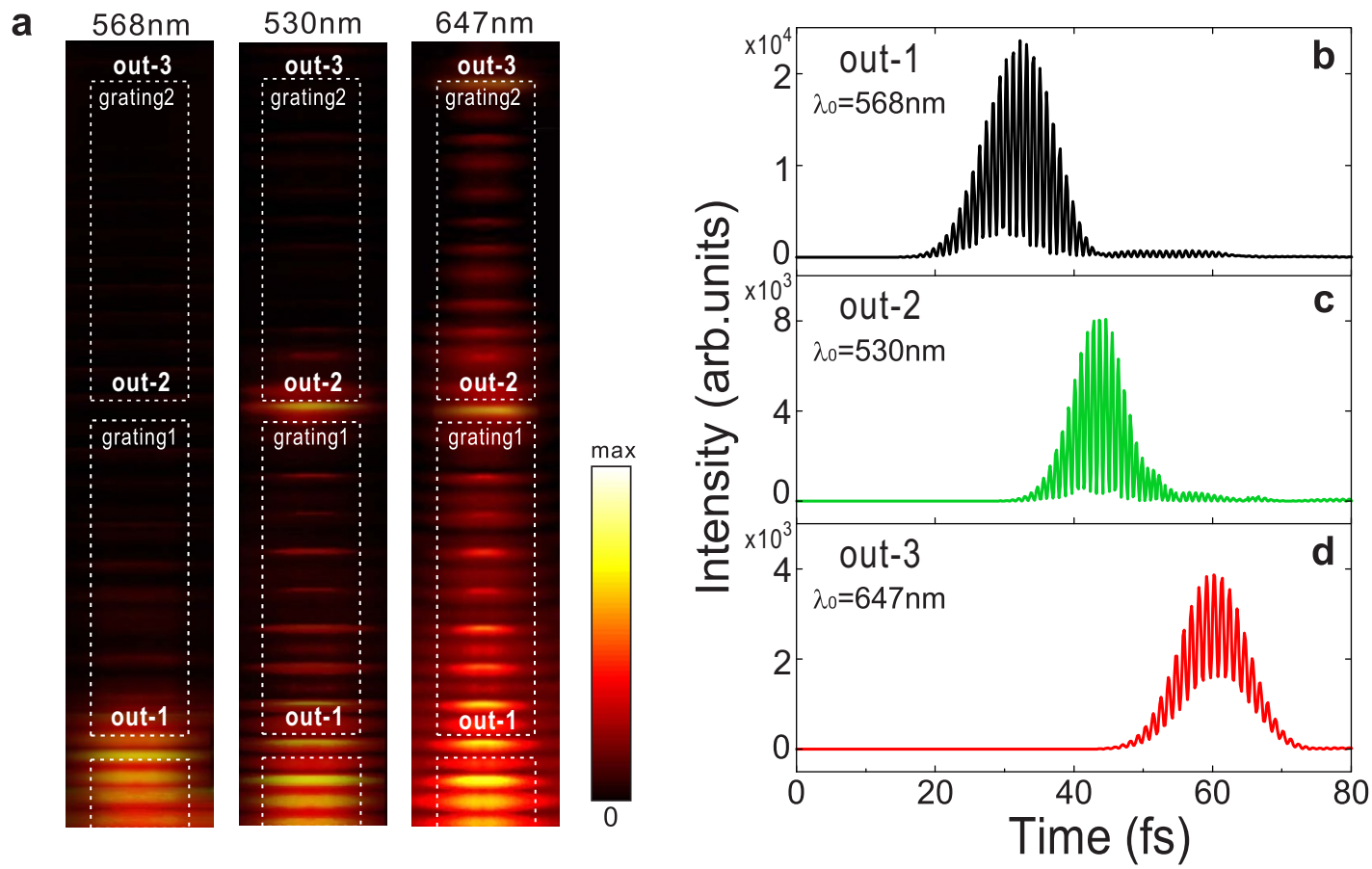

Figure $4 \mid$ The FDTD simulation of the transport process of SPs on the structured nanowire of Fig. 3. (a). The calculated electric field distributions $\left(|\mathbf{E}|^{2}\right)$ along the nanowire (diameter $170 \mathrm{~nm}$ ) for incidence wavelengths of $568 \mathrm{~nm}$ (left), $530 \mathrm{~nm}$ (middle) and $647 \mathrm{~nm}$ (right), respectively. The white dash boxes indicate the corrugation gratings. The gap between neighboring dashed boxes corresponds to a "bus stop" groove. (b-d) The calculated temporal spectra of the output light pulses for wavelengths $568 \mathrm{~nm}, 530 \mathrm{~nm}$ and $647 \mathrm{~nm}$, respectively. Different colors are released chronologically at $15-$ femtosecond-or-so intervals. In the simulation the incident laser pulse is assumed to be a focused Gaussian beam with a pulse length of $2 \mathrm{fs}$ and a central wavelength $568 \mathrm{~nm}, 530 \mathrm{~nm}$ and $647 \mathrm{~nm}$, respectively. 
silicon. Second, the plasmonic PSS (based on a $170 \mathrm{~nm}$-diameter silver wire, for example) can achieve ten-times more efficient optical confinement because it possess a much smaller mode area $\left(0.06 A_{0}\right.$, $\left.A_{0}=\lambda^{2} / 4\right)$ comparing with the non-plasmonic waveguides for visible wavelengths. Third and more importantly, the plasmonic PSS can be fully compatible with current CMOS techniques, which makes it possible to develop on-chip photonic devices and to integrate nanophotonics with microelectronics.

Furthermore, based on corrugated silver nanowires on silicon chip, we can construct plasmonic networks to perform two-dimensional (2D) position-sensitive spectral splitting. Figure 5a schematically illustrates such a cross network, which consists of four branches of silver nanowires (170 nm diameter) with different corrugation periodicities of $520 \mathrm{~nm}, 560 \mathrm{~nm}, 470 \mathrm{~nm}$, and $440 \mathrm{~nm}$, respectively.
When a white beam is incident on the center of the cross, the electricfield distributions $\left(|\mathbf{E}|^{2}\right)$ of the SPs propagating in the network have been numerically calculated at wavelengths $568 \mathrm{~nm}$ (yellow), $530 \mathrm{~nm}$ (green), and $488 \mathrm{~nm}$ (blue), and $647 \mathrm{~nm}$ (red), respectively. The calculations demonstrate that the yellow beam propagates to the "North" (Fig. 5b), the blue beam propagates to the "East" (Fig. 5c), the green beam propagates to both the "West" and "South" (Fig. 5d), and the red beam can reach all directions (Fig. 5e). With the 2D scheme, therefore, parallel demultiplexing and processing of light signals can be realized. In addition, the pixels can be flexibly tuned in two dimensions for spectral splitting and imaging.

In summary, by fabricating corrugated silver nanowires on silicon wafer, we have realized for the first time nanoscale position-sensitive spectral splitting of photons as well as time-of-flight monitoring of

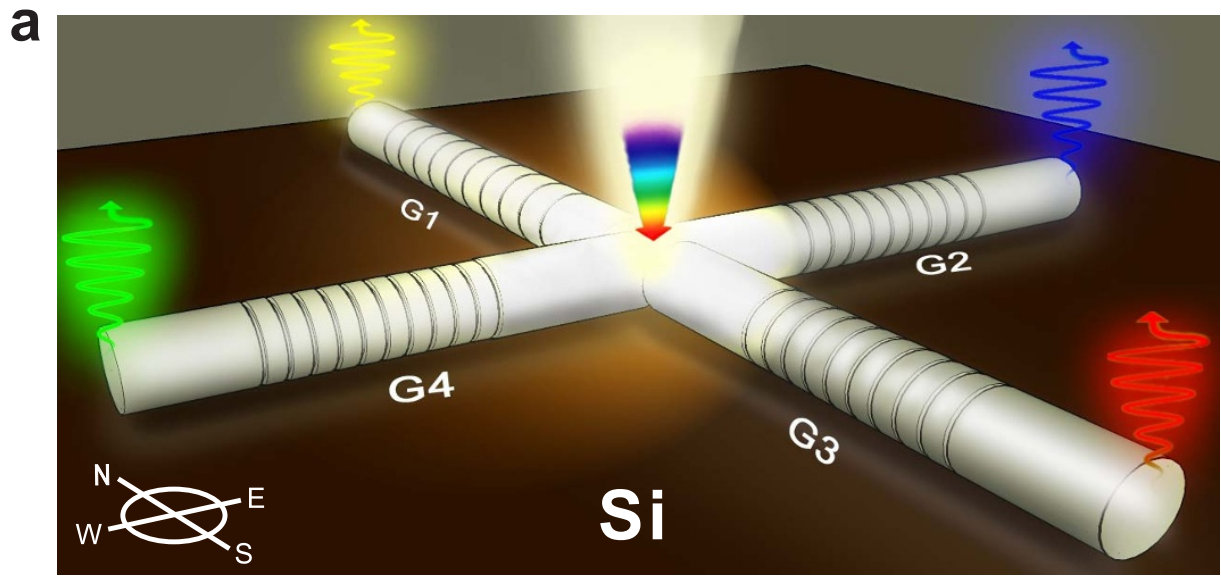

b

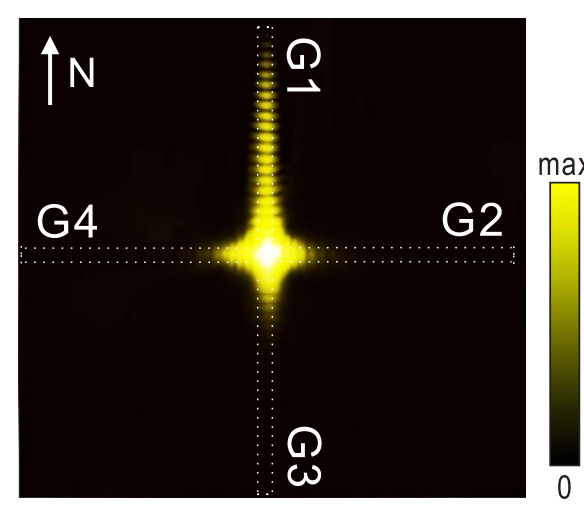

C

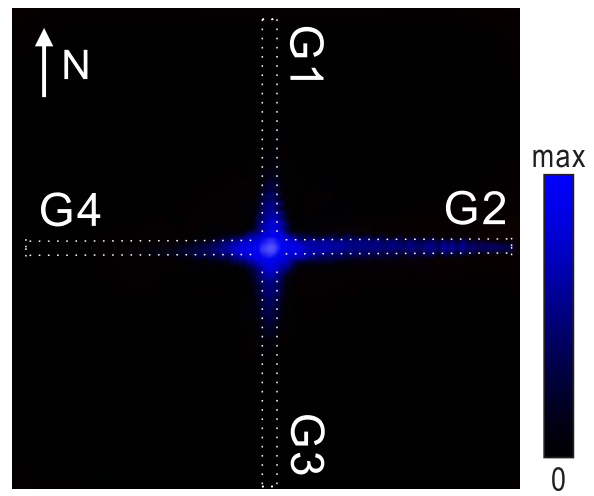

d

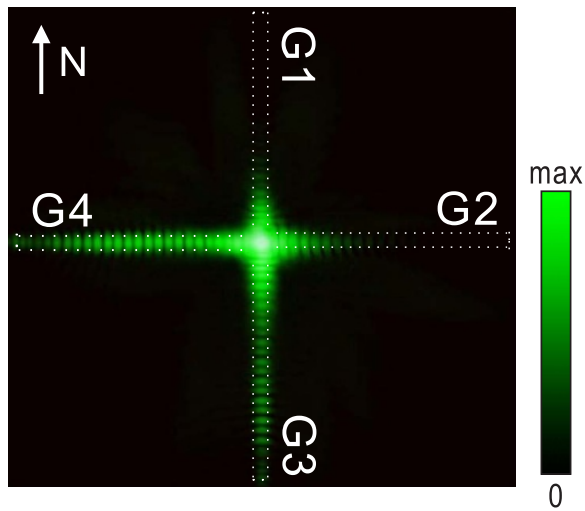

e

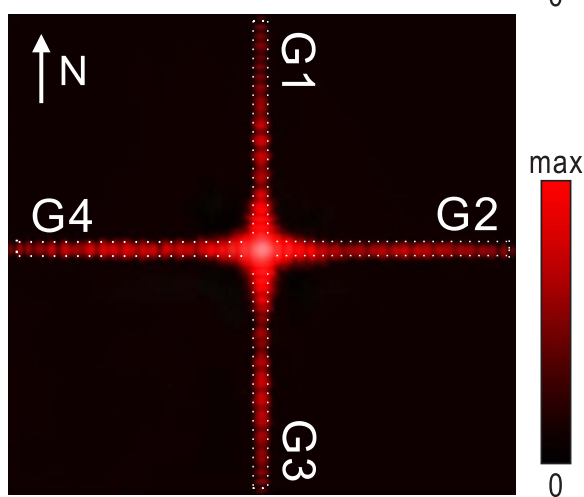

Figure 5 The FDTD simulation of a 2D PSS with structured cross nanowire networks. (a). Schematic of the 2D network consisting of four silver nanowire arms with different corrugation periods: $520 \mathrm{~nm}(\mathrm{G} 1), 560 \mathrm{~nm}(\mathrm{G} 2), 470 \mathrm{~nm}$ (G3) and $440 \mathrm{~nm}$ (G4), respectively. The wire diameter is always fixed at $170 \mathrm{~nm}$, and the width and depth of the corrugations are both set at $30 \mathrm{~nm}$. Each grating consists of eight corrugations, starting from the location $3 \mu \mathrm{m}$ away from the center cross. (b-e). The calculated electric-field distributions $\left(|\mathbf{E}|^{2}\right)$ of the SPs propagating in the network with wavelengths $568 \mathrm{~nm}$, $488 \mathrm{~nm}, 530 \mathrm{~nm}$, and $647 \mathrm{~nm}$, respectively. The arrow " $\mathrm{N}$ " indicates the north direction. In the simulation, the incident light source is an unpolarized Gaussian beam focused on the central cross. When a white light incidents at the center cross, SPs with specific colors propagate along different channels. 
femtosecond optical pulses. Moreover, the corrugated nanowires can be constructed into compact $2 \mathrm{D}$ spatiotemporal-sensitive networks. Our studies provide a new approach to develop spatiotemporalresolved spectral splitting networks on nanoscales. Such devices may also be applied for optical frequency division and optical signal demultiplexing on nanoscales. Most significantly, the plasmonic devices are ultracompact on silicon wafers and the nanofabrication process is fully compatible with current CMOS techniques, which demonstrates the feasibility of design and fabrication of on-chip nanophotonics combined with microelectronics. The development of spatiotemporal optical signal processing on the nanoscales together with other recent achievements (e.g., nanophotonic logics and networks ${ }^{31,53,54}$ ) could make plasmonic circuits more reality for the next generation information technology in the future.

\section{Methods}

Sample fabrication. The silver nanowires are chemically synthesized ${ }^{55}$ with diameter $170 \mathrm{~nm}$ and length around $15 \mu \mathrm{m}$. The wires are then transferred onto a polished silicon substrate, followed by a coating of $5 \mathrm{~nm}$-thick layer of $\mathrm{SiO}_{2}$ via magnetron sputtering. The corrugations are fabricated on the silver nanowires by focus-ionbeam milling (FIB, Helios Nanolab 600i). Both the width and the depth of the corrugation are fixed at $30 \mathrm{~nm}$, and the spatial periodicity varies from $400 \mathrm{~nm}$ to $600 \mathrm{~nm}$. The profile of the "bus stop" groove between different sections of corrugations is optimized in order to increase the coupling efficiency between the incident light and SPs.

Experimental setup. As schematically shown in Supplementary Fig. S2, the input polychromatic laser beam (with main wavelengths $647 \mathrm{~nm}, 568 \mathrm{~nm}, 530 \mathrm{~nm}$, $520 \mathrm{~nm}, 514 \mathrm{~nm}, 488 \mathrm{~nm}$ from Spectra-Physics Lasers, 2018-RM) is focused by an objective $(100 \times)$ to the end or the middle of the nanowire. The emitted light from the wires is analyzed by a spectrometer (Princeton Instruments,SP-2500). The emission is also imaged by a CCD. The spectrum measurement and the emission imaging are switchable by a mirror.

Simulation. Based on the full-wave finite-difference time-domain (FDTD) method $^{56}$ we carry out the numerical simulation on the transportation of SPs and the scattered light spectra on the structured silver nanowires by using a commercial software package (Lumerical, FDTD_Solutions version 8.0.1). The geometry is modeled as that of the measured samples. The simulation is modeled with optimum parameters of focused Gaussian sources and detector positions ${ }^{57}$.

1. Almeida, V. R. et al. All-optical control of light on a silicon chip. Nature 431, 1081-1084 (2004).

2. Levenson, R. M. \& Hoyt, C. C. Spectral imaging and microscopy. Am. Lab. 32, 26-34 (2000).

3. Cerezo, A., Godfrey, T. J. \& Smith, G. D. W. Rev. Sci. Instrum. Application of a position sensitive detector to atom probe microanalysis. Rev. Sci. Instrum. 59, 862-866 (1988).

4. Keil, R. et al. All-optical routing and switching for three-dimensional photonic circuitry. Sci. Rep. 1, 94 (2011).

5. Vikram, C. S. \& Caulfield, H. J. Position-sensing detector for logical operations using incoherent light. Opt. Eng. 44, 115201 (2005).

6. Gys, T., D'Ambrosio, C., Leutz, H., Piedigrossi, D. \& Puertolas, D. A new positionsensitive photon detector based on an imaging silicon pixel array (ISPA-tube). Nuclear. Instr. and Meth. in Phys. Res. A 355, 386-389 (1995).

7. Harvey, A. R., Baele, J., Greenaway, A. H., Hanlon, T. J. \& Williams, J. Technology options for imaging spectrometry. Proc. SPIE 4132, 13-24 (2000).

8. Colinge, J. P. Silicon-on-Insulator Technology: Materials to VLSI, Kluwer Academic Publishers, Dordrecht, Netherlands (1991).

9. Shahidi, G. G. SOI technology for the GHz era. IBM J. RES. \& DEV. 46, 121-131 (2002).

10. Jaju, V. \& Dalal, V. Silicon-on-Insulator Technology. EE 530, Advances in MOSFETs, Spring (2004).

11. Taillaert, D., Bienstman, P. \& Baets, R. Compact efficient broadband grating coupler for silicon-on-insulator waveguides. Opt. Lett. 29, 2749-2751 (2004)

12. Hattasan, N. et al. High-efficiency SOI fiber-to-chip grating couplers and low-loss waveguides for the short-wave infrared. IEEE Photonic Technol. Lett. 24, 1536-1538 (2012).

13. Ang, T. W. et al. Grating couplers using silicon-on-insulator. SPIE 3620, 79-86 (1999)

14. Trinh, P. D. et al. Silicon-on-insulator (SOI) phased-array wavelength multi/ demultiplexer with extremely low-polarization sensitivity. IEEE Photonic Technol. Lett. 9, 940-942 (1997).

15. Brouckaert, J. et al. Planar concave grating demultiplexer with high reflective Bragg reflector facets. IEEE Photonic Technol. Lett. 20, 309-311 (2008).

16. Dionne, J. A. et al. Silicon-based plasmonics for on-chip photonics. IEEE J. Sel. Top. Quant. Electron. 16, 295-306 (2010).
17. Schuller, J. A. et al. Plasmonics for extreme light concentration and manipulation. Nat. Mater. 9, 193-204 (2010).

18. Fang, N., Lee, H., Sun, C. \& Zhang, X. Sub-diffraction-limited optical imaging with a silver superlens. Science 308, 534-537 (2005).

19. Bao, Y. J., Peng, R. W., Shu, D. J. et al. Role of interference between localized and propagating surface waves on the extraordinary optical transmission through a subwavelength-aperture array. Phys. Rev. Lett. 101, 087401 (2008).

20. Barnes, W. L., Dereux, A. \& Ebbesen, T. W. Surface plasmon subwavelength optics. Nature 424, 824-830 (2003).

21. Ozbay, E. Plasmonics: merging photonics and electronics at nanoscale dimensions. Science 311, 189-193 (2006).

22. Genet, C. \& Ebbesen, T. W. Light in tiny holes. Nature 445, 39-46 (2007).

23. Gramotnev, D. K. \& Bozhevolnyi, S. I. Plasmonics beyond the diffraction limit. Nat. Photon. 4, 83-91 (2010).

24. Zia, R., Schuller, J. A., Chandran, A. \& Brongersma, M. L. Plasmonics: the next chip-scale technology. Mater. Today 9, 20-27 (2006).

25. Bozhevolnyi, S. I. Plamonic nanoguides and circuits (Pan Stanford Publishing Pte. Ltd. 2009).

26. Engheta, N. Circuits with light at nanoscales: optical nanocircuits inspired by metamaterials. Science 317, 1698-1702 (2007)

27. Schnell, M. et al. Controlling the near-field oscillations of loaded plasmonic nanoantennas. Nat. Photon. 3, 287-291 (2009).

28. Zhang, Z. J. et al. Plasmonic antenna array at optical frequency made by nanoapertures. Appl. Phys. Lett. 93, 171110 (2008).

29. Liu, J. S. Q. et al. A submicron plasmonic dichroic splitter. Nat. Commun. 2, 525 (2011).

30. Laux, E. et al. Plasmonic photon sorters for spectral and polarimetric imaging. Nat. Photon. 2, 161-164 (2008)

31. Wei, H. et al. Cascaded logic gates in nanophotonic plasmon networks. Nat Commun. 2, 387 (2011).

32. Maier, S. A. Plasmonics: fundamentals and applications (Springer, 2007).

33. Genov, D. A., Ambati, M. \& Zhang, X. Surface plasmon amplification in planar metal films. IEEE J. Quant. Electron. 43, 1104-1108 (2007).

34. Oulton, R. F., Sorger, V. J., Genov, D. A., Pile, D. F. P. \& Zhang, X. A hybrid plasmonic waveguide for subwavelength confinement and long-range propagation. Nat. Photon. 2, 496-500 (2008).

35. Chu, H.-S., Li, E.-P., Bai, P. \& Hedge, R. Optical performance of single-mode hybrid dielectric-loaded plasmonic waveguide-based components. Appl. Phys. Lett. 96, 221103 (2010)

36. Sorger, V. J. et al. Experimental demonstration of low-loss optical waveguiding at deep sub-wavelength scales. Nat. Commun. 2, 331 (2011).

37. Krasavin, A. V. \& Zayats, A. V. Silicon-based plasmonic waveguides. Opt. Express 18, 11791-11799 (2010)

38. Krasavin, A. V. \& Zayats, A. V. Three-dimensional numerical modeling of photonic integration with dielectric-loaded SPP waveguides. Phys. Rev. B 78, 045425 (2008).

39. Lee, J. et al. Time-of-flight measurement with femtosecond light pulses. Nat. Photon. 4, 716-720 (2010).

40. Zhang, H. F., Maslov, K., Stoica, G. \& Wang, L. V. Functional photoacoustic microscopy for high-resolution and noninvasive in vivo imaging. Nat. Biotechnol. 24, 848-851 (2006)

41. Feigenbaum, E. \& Atwater, H. A. Resonant guided wave networks. Phys. Rev. Lett. 104, $147402(2010)$

42. Li, Q. \& Qiu, M. Plasmonic wave propagation in silver nanowires: guiding modes or not? Opt. Express 21, 8587-8595 (2013).

43. Zou, C. L. et al. Plasmon modes of silver nanowire on a silica substrate. Appl. Phys. Lett. 97, 183102 (2010).

44. Ditlbacher, H. et al. Silver nanowire as surface plasmon resonators. Phys. Rev. Lett. 95, 257403 (2005)

45. Sanders, A. W. et al. Observation of plasmon propagation, redirection, and fanout in silver nanowires. Nano Lett. 6, 1822-1826 (2006).

46. Li, Z. P. et al. Effect of a proximal substrate on plasmon propagation in silver nanowires. Phys. Rev. B 82, 241402 (2010).

47. Wei, H. et al. Highly tunable propagating surface plasmons on supported silver nanowires. PNAS. 110, 4494-4499 (2013).

48. Nguyen, H. G. et al. All optical up-coversion of WLAN signal in $60 \mathrm{GHz}$ range with side-band suppression. IEEE Radio and Wireless Symposium 590-593 (2009).

49. Frankel, M. Y. \& Esman, R. D. Optical single-sideband suppressed-carrier modulator for wide-band signal processing. J. Lightwave Technol. 16, 859-863 (1998).

50. Vermeulen, D. et al. High-efficiency fiber-to-chip grating couplers realized using an advanced CMOS-compatible silicon-on-insulator platform. Opt. Express 18, 18278-18283 (2010).

51. Xiao, Z. et al. Design for broadband high-efficiency grating couplers. Opt. Lett. 37, 530-532 (2012).

52. Li, Y. et al. Compact grating couplers on silicon-on-insulator with reduced backreflection. Opt. Lett. 37, 4356-4358 (2012).

53. Pyayt, A. L. et al. Integration of photonic and silver nanowire plasmonic waveguides. Nat. Nanotechnol. 3, 660-665 (2008).

54. Li, Y. et al. Nanowire electronic and optoelectronic devices. Mater. Today $9,18-27$ (2006). 
55. Sun, Y., Mayers, B., Herricks, T. \& Xia, Y. Polyol Synthesis of Uniform Silver Nanowires: A Plausible Growth Mechanism and the Supporting Evidence. Nano Lett. 3, 955-960 (2003).

56. Taflove, A. \& Hagness, S. C. Computational electrodynamics: the finite-difference time-domain method. Second Edition.

57. Laroche, T. \& Girard, C. Near-field optical properties of single plasmonic nanowires. Appl. Phys. Lett. 89, 233119 (2006).

\section{Acknowledgements}

This work was supported by the Ministry of Science and Technology of China (Grant Nos. 2012CB921502 and 2010CB630705), the National Science Foundation of China (Grant Nos. 11034005, 61077023, and 11021403), and partly by the Ministry of Education of China (20100091110029). XRH was supported by the U.S. Department of Energy, Office of Science, Office of Basic Energy Sciences, under Contract No. DE-AC02-06CH11357. NXF acknowledges partial support by NSF Grant no. CMMI-1120724.

\section{Author contributions}

R.W.P. and Q.H. developed the concept. Q.H., R.H.F., D.H.X. and Q.J.W. fabricated the samples. Q.H. characterized the samples. Q.H. and D.H.X. performed the measurements. Q.H. and Y.Z. performed the calculations. Q.H., R.W.P., N.X.F., M.W. and X.R.H. analyzed and interpreted the data and implications. Q.H., R.W.P., X.R.H. and M.W. wrote the manuscript. R.W.P. and M.W. supervised the researches.

\section{Additional information}

Supplementary information accompanies this paper at http://www.nature.com/ scientificreports

Competing financial interests: The authors declare no competing financial interests.

How to cite this article: $\mathrm{Hu}$, Q. et al. Position-sensitive spectral splitting with a plasmonic nanowire on silicon chip. Sci. Rep. 3, 3095; DOI:10.1038/srep03095 (2013).

(c) (1) $(9)$ This work is licensed under a Creative Commons Attribution-

BY NG ND NonCommercial-NoDerivs 3.0 Unported license. To view a copy of this license, visit http://creativecommons.org/licenses/by-nc-nd/3.0 